\title{
Spontaneous breathing anesthesia for cervical tracheal resection and reconstruction
}

\author{
Yanran Zhou ${ }^{1 \#}$, Hui Liu ${ }^{1 \#}, \mathrm{Xi} \mathrm{Wu}^{1}$, Shuben $\mathrm{Li}^{2}$, Lixia Liang ${ }^{1}$, Qinglong Dong ${ }^{1}$ \\ ${ }^{1}$ Department of Anesthesiology, ${ }^{2}$ Department of Cardiothoracic Surgery, The First Affiliated Hospital of Guangzhou Medical University, Guangzhou \\ 510120, China \\ Contributions: (I) Conception and design: Y Zhou, H Liu, Q Dong; (II) Administrative support: Q Dong; (III) Provision of study materials or patients: \\ Y Zhou, H Liu, X Wu, S Li, L Liang; (IV) Collection and assembly of data: Y Zhou, X Wu; (V) Data analysis and interpretation: Y Zhou, H Liu, \\ $\mathrm{X} \mathrm{Wu}$; (VI) Manuscript writing: All authors; (VII) Final approval of manuscript: All authors. \\ \#These authors contributed equally to this work. \\ Correspondence to: Qinglong Dong. Department of Anesthesiology, the First Affiliated Hospital of Guangzhou Medical University, No. 151, Yanjiang \\ Road, Guangzhou 510120, China. Email: 335025746@qq.com.
}

Background: Spontaneous breathing anesthesia (SBA) may have advantages over general anesthesia for cervical tracheal resection and reconstruction (TRR), avoiding the difficulties and complication caused by endotracheal intubation and surgical cross-field intubation. This prospective study evaluates SBA for cervical TRR.

Methods: Date was obtained from 35 patients who had cervical TRR under SBA from May 2015 to March 2019. Intravenous sedation and ultrasound-guided bilateral superficial cervical plexus block (CPB) were applied to maintain effective analgesia and sedation.

Results: Thirty-two patients with tracheal tumors and 3 patients with post-intubation tracheal stenosis underwent TRR. After the airway was opened, 29 patients resumed stable spontaneous breathing, 1 patient needed high-frequency jet ventilation, and 1 patient needed anesthesia conversion for surgical reasons.

Conclusions: Spontaneous breathing anesthesia is feasible for the cervical TRR. It can be an alternative anesthetic technique for certain patients.

Keywords: Spontaneous breathing anesthesia (SBA); tracheal resection and reconstruction (TRR); cervical plexus block (CPB)

Submitted Jul 29, 2019. Accepted for publication Nov 19, 2019.

doi: $10.21037 /$ jtd.2019.11.70

View this article at: http://dx.doi.org/10.21037/jtd.2019.11.70

\section{Introduction}

Tracheal resection and reconstruction (TRR) is a challenging procedure for anesthesiologists and surgeons because they share the airway for procedure and ventilation at the same time. The conventional airway management for TRR is endotracheal intubation and surgical crossfield intubation under general anesthesia. However, the endotracheal intubation and surgical cross-field intubation could cause complications in anesthesia induction, recovery and surgical anastomosis.

Recently, it has been reported that by comparison with general anesthesia, spontaneous breathing anesthesia (SBA) for video-assisted thoracoscopic surgery (VATS) can reduces the adverse effects in hemodynamics, ventilator associated lung injury (VALI) and lymphocyte responses $(1,2)$. In this study, we describe and evaluate the feasibility of SAB for cervical TRR.

\section{Methods}

\section{Subjects}

This is a prospective study consist of patients who had 

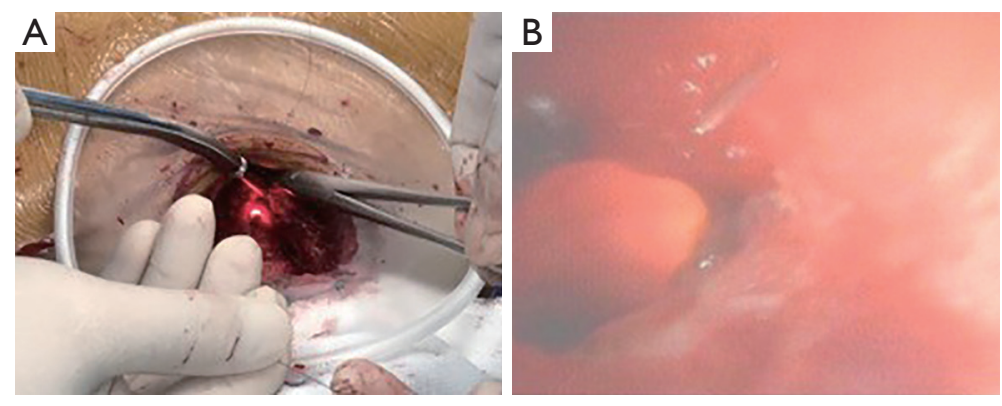

Figure 1 Confirm the location of the lesion. (A) The surgeon observed the lightest spot of the bronchoscope through the tracheal wall; (B) the anesthesiologist observed the needle through the bronchoscopy.

cervical TRR under SBA from May 2015 to March 2019. All patients meet the indications for SBA, including: ASA grade of I-III, and a body mass index (BMI) $<26 \mathrm{~kg} / \mathrm{m}^{2}$. Patients with a blood disorders, disorders related to constrained cardiac output (such as mitral stenosis, aortic stenosis, severe arrhythmias, hypertrophic cardiomyopathy), or contraindicated for superficial cervical plexus block (CPB) were ineligible for this procedure.

All patients had flexible bronchoscopy to evaluate the location and extent of lesion, as well as the pathology. If bronchoscopic assessment revealed more than $60-70 \%$ obstruction of the upper airway, the bronchoscopic balloon dilatation or bronchoscopic electrocautery was applied to reduce airway obstruction. Preoperative evaluation including detailed clinical information, laboratory tests, high-resolution computerized tomography (HRCT), electrocardiogram, ultrasonic cardiogram, lung function test, artery blood gas analysis.

\section{Anestbetic management}

\section{Anesthesia}

All patients were premedicated with atropine $0.5 \mathrm{mg}$ and dexmedetomidine $1 \mu \mathrm{g} \cdot \mathrm{kg}^{-1} \cdot \mathrm{h}^{-1}$ for 15 minutes. For induction, propofol 3-4 $\mu \mathrm{g} \cdot \mathrm{mL}^{-1}$ target-controlled infusion (TCI) and sufentanil $0.1-0.15 \mu \mathrm{g} \cdot \mathrm{kg}^{-1}$. Inserted laryngeal mask airway (LMA) when BIS below 60, using simultaneous intermittent mandatory ventilation (SIMV). Inserted gastric tube via LMA. After induction, ultrasound-guided bilateral superficial CPB was done with a $22 \mathrm{G}$ needle, using $10 \mathrm{~mL}$ of $0.375 \%$ ropivacaine on each side. Anesthesia maintain with dexmedetomidine $0.5-1.0 \mu \mathrm{g} \cdot \mathrm{kg}^{-1} \cdot \mathrm{h}^{-1}$, remifentanil $0.03-0.08 \mu \mathrm{g} \cdot \mathrm{kg}^{-1} \mathrm{~min}^{-1}$, propofol (TCI) $1.0-2.0 \mu \mathrm{g} \cdot \mathrm{mL}^{-1}$. BIS maintain between 40-60. Before the airway was opened, reduced the administration rate of propofol and remifentanil, changed to manual assisted ventilation, $\mathrm{FiO}_{2} 1$, air flow $2-5 \mathrm{~L} / \mathrm{min}$.

\section{Confirm the location and extent of the lesion}

Three patients with tracheal stenosis were confirmed the location and extent of the lesion with flexible bronchoscopy, and 32 patients were confirmed with fluorescent bronchoscopy. Before bronchoscopy, larynx and airway surface anesthesia with $2 \%$ lidocaine was applied. The anesthesiologist used flexible bronchoscopy to locate the lower margin of the lesion through the conduit of LMA. The surgeon observed the lightest spot of the bronchoscope through the tracheal wall (Figure $1 A$ ), used a $5 \mathrm{~mL}$ syringe needle to penetrate into the tracheal wall at the brightest spot. Then, the anesthesiologist observed the needle through the bronchoscopy, accurately confirmed the lower margin that need to be removed (Figure 1B). The locating method of upper margin of lesion was the same as above.

\section{Airway management}

Approximately 15 minutes before the airway was opened, changed the ventilation mode from SIMV to manual assisted ventilation $\left(\mathrm{FiO}_{2} 1\right.$, air flow $\left.2-5 \mathrm{~L} / \mathrm{min}\right)$. The patients were induced to resume spontaneous breathing. After the trachea was incised, a sterile catheter was inserted in the distal portion of the trachea across the surgical field, oxygen was continuously supplied to distal airway (air flow 2-5 L/min) (Figure $2 A$ ). If the $\mathrm{SpO}_{2}$ decreased to $90 \%$, connected the catheter to the jet ventilation circuit for jet ventilation (driving pressure $=0.5-1.0 \mathrm{bar}, \mathrm{FiO}_{2}=1.0$, respiration rate $=60$ breaths $/ \mathrm{min}, \mathrm{I}: \mathrm{E}$ ratio of $1: 1$ ) (Figure $2 B$ ). If there was no obvious improvement of $\mathrm{SpO}_{2}$, the distal trachea was intubated with a sterile endotracheal tube, and 

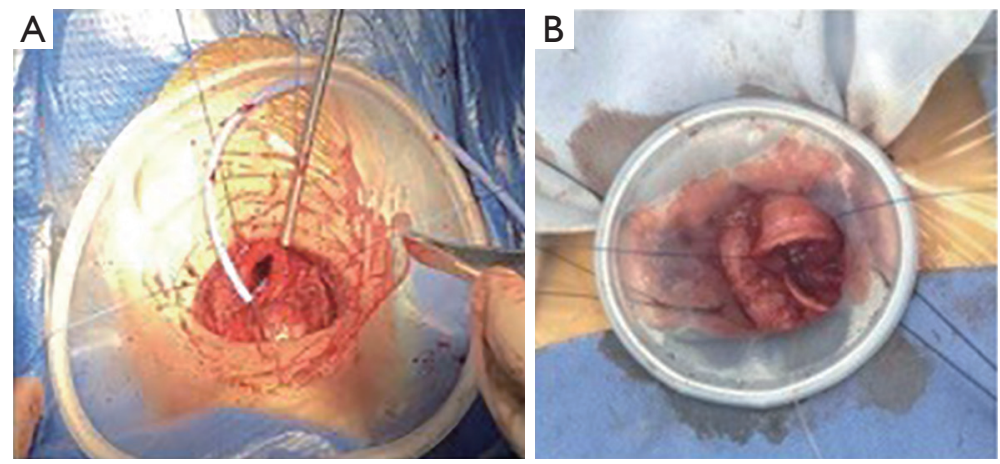

Figure 2 Airway management. (A) A sterile catheter was inserted in the distal portion of the trachea to supply oxygen; (B) a sterile catheter was re-moved from the distal trachea.

connected to a breathing circuit for SIMV ventilation.

After the anastomosis was completed, the sterile catheter/jet ventilation catheter/endotracheal tube was removed from the surgical field. The anastomosis is checked by fiberoptic bronchoscopy, then a air leak test was performed to ensure that the anastomosis was leak proof. To the patients with intubation after airway opened, unstable spontaneous breathing or the latest $\mathrm{PaCO}_{2}>50 \mathrm{mmHg}$, changed ventilation mode to SIMV after the anastomosis was completed. After the operation, removed the LMA after patients had stable spontaneous breathing and recovered consciousness.

\section{Anesthesia monitors}

During the procedure, heart rate (HR), electrocardiography (ECG), pulse oxygen saturation $\left(\mathrm{SpO}_{2}\right)$, bispectral index (BIS), invasive arterial blood pressure (ABP) were continuously monitored. End-tidal $\mathrm{CO}_{2}$ pressure $\left(\mathrm{E}_{\mathrm{T}} \mathrm{CO}_{2}\right)$ and fraction of inspiration $\mathrm{O}_{2}\left(\mathrm{FiO}_{2}\right)$ were continuously monitored before the airway was opened and after airway anastomosis completed. Arterial blood gas (ABG) was intermittently monitored during the procedure. $\mathrm{PaO}_{2}$ and $\mathrm{PaCO}_{2}$ after anesthesia induction were monitored 20 minutes after anesthesia induction.

Data were analysed using SPSS 19.0 software (SPSS Inc., Chicago, IL, USA). Normal distribution data is expressed as mean \pm standard deviation and categorical data as number (proportion). Comparisons of the differences between values at a specific time $\mathrm{PaO}_{2}$ and $\mathrm{PaCO}_{2}$ were using oneway ANOVA. Statistical significance was presented as a $\mathrm{P}$ value of $<0.05$ throughout the study.

\section{Results}

A total of 35 patients were enrolled in the study. The age, gender, ASA, BMI, and preoperative treatment are shown in Table 1. The intraoperative conditions and postoperative hospital stay days are shown in Table 2.

All patients resumed to spontaneous breathing before the airway was opened. After the airway was opened, a sterile catheter was inserted in the distal portion of the trachea to supply oxygen (Figure $2 A$ ). Twenty-nine cases (83\%) maintained $\mathrm{SpO}_{2}>90 \%$. During the anastomosis phase of procedure, the sterile catheter was removed from the distal trachea after discussion between surgeons and anesthesiologist in 4 cases (11\%), because of the obstruction of surgical field (Figure $2 \mathrm{~B}$ ). One case (3\%) showed a $\mathrm{SpO}_{2}$ decrease to $85 \%$ after the airway was opened, connected the catheter to high-frequency ventilation circuit for highfrequency ventilation. The $\mathrm{SpO}_{2}$ was suddenly declined in one patient, diagnosed as right pleural rupture leading to the right pneumothorax, immediately intubated across the field. After expanded the lung and sutured the right ruptured pleura, pulled out the endotracheal tube, the patient resumed to spontaneous breathing, the operation was smooth (Table 3).

The patients showed an arterial oxygen concentration $\left(\mathrm{PaO}_{2}\right)$ of $396.5 \pm 83.8 \mathrm{mmHg}$ after anesthesia induction. During the open airway phase, $\mathrm{PaO}_{2}$ decreased to $173.4 \pm$ $53.6 \mathrm{mmHg}$, and then recovered to $279.1 \pm 82.7 \mathrm{mmHg}$ after the airway closed. After anesthesia induction, the patients showed an arterial carbon dioxide concentration $\left(\mathrm{PaCO}_{2}\right)$ of $49.3 \pm 6.0 \mathrm{mmHg}$. Before the airway was opened, the $\mathrm{PaCO}_{2}$ reached to $53.9 \pm 8.1 \mathrm{mmHg}$, then slightly decreased to $51.0 \pm 6.6 \mathrm{mmHg}$ during the open airway 
Table 1 Patient characteristics

\begin{tabular}{|c|c|}
\hline Variables & Value \\
\hline Age (yr) & $40 \pm 17$ \\
\hline \multicolumn{2}{|l|}{ Gender } \\
\hline Male & 19 \\
\hline Female & 16 \\
\hline \multicolumn{2}{|l|}{ ASAl } \\
\hline I & 4 \\
\hline II & 26 \\
\hline III & 5 \\
\hline BMI $\left(\mathrm{kg} / \mathrm{m}^{2}\right)$ & $20.8 \pm 3.5$ \\
\hline \multicolumn{2}{|l|}{ Preoperative treatment } \\
\hline Bronchoscopic balloon dilatation & 3 \\
\hline Bronchoscopic electrocautery & 26 \\
\hline No preoperative treatment & 6 \\
\hline \multicolumn{2}{|l|}{ Pathological diagnosis } \\
\hline Adenoid cystic carcinoma & 21 \\
\hline Squamous-cell carcinoma & 7 \\
\hline Inflammatory myofibroblastic tumor & 1 \\
\hline Glomangioma & 1 \\
\hline Cicatricial tissue & 3 \\
\hline Others & 2 \\
\hline
\end{tabular}

Table 2 Intraoperative details and postoperative hospital stay

\begin{tabular}{lc}
\hline Variables & Value \\
\hline Length of excision (cm) & $3.2 \pm 0.3$ \\
Surgery duration (min) & $178.2 \pm 74.1$ \\
Anesthesia duration (min) & $252.1 \pm 69.3$ \\
Time of tracheal end-to-end anastomosis (min) & $42.0 \pm 17.7$ \\
Anesthesia recovery time (min) & $17.3 \pm 8.2$ \\
Postoperative hospital stay $(\mathrm{d})$ & $9.3 \pm 0.9$ \\
\hline
\end{tabular}

phase. After the anastomosis was completed, the $\mathrm{PaCO}_{2}$ was $51.6 \pm 7.6 \mathrm{mmHg}$ (Figure 3).

Stop anesthetic infusion after the operation. All patients resumed stable spontaneous breathing and removed the LMA after recovered consciousness.

\section{Discussion}

The most common etiology of cervical TRR is tracheal tumors, post-intubation tracheal stenosis, trauma and inflammation (3). The conventional airway management in cervical TRR is endotracheal intubation and surgical crossfield intubation (4).

For the patients with tracheal tumors, muscle relaxation after anesthesia induction may lead to tracheal collapse and an aggravation of the airway obstruction, resulting in life-threatening hypoxemia. Therefore, awaked fiberoptic intubation in emergency situation has also been reported (5). However, when the tracheal tumor is close to the glottis, the endotracheal intubation may lead to tumor shedding, bleeding, especially pedicled tumors and hemangioma. It has been reported that inserted LMA after anesthesia induction to avoid the possibility of injury to the tumor by endotracheal intubation (6). For patients with benign tracheal stenosis, it is often characterized by the mucosa chronic inflammation with necrosis and granulocytic infiltration, formation of scar tissue. Due to the poor expansibility of tracheal membrane, if endotracheal intubation fails, it may lead to swelling and bleeding of the lesion, reintubation become more difficult (7).

The safety and feasibility of SBA for VATS surgery have been reported both in our experience and recent studies (8-10). Based on our experience of SBA and surgery management, it allowed us to perform this anesthetic technique for cervical TRR.

Effective analgesia is one of the main concerns of SBA. According to different procedures, thoracic paravertebral nerve block or intercostal nerve block can provide effective analgesia during VATS procedure on lung. In the cervical TRR, we used ultrasound-guided bilateral superficial $\mathrm{CPB}$ and a continuous infusion of low dosage of remifentanil $(0.03-0.05 \mu \mathrm{g} / \mathrm{kg} / \mathrm{min})$ to provide effective analgesia. It's reported that cervical epidural block and sedation for cervical TRR by Macchiarini (11). Although the complications and adverse effects following cervical epidural block were not observed, however, due to its fatal complications, we replaced with bilateral superficial CPB provided similar effective analgesia. There was no restlessness or hemodynamic instability due to inadequate analgesia. Superficial CPB has been used for various head and neck surgeries intra- and postoperative analgesic management. Studies reported that if the local anesthetic spread to the deep cervical plexus might lead to phrenic 
Table 3 Airway management during airway opened

\begin{tabular}{lc}
\hline Variables & $\mathrm{N}=35$ \\
\hline Spontaneous breathing (a catheter was removed from the trachea) & $4(11 \%)$ \\
Spontaneous breathing (a catheter was inserted in the distal portion of the trachea) & $29(83 \%)$ \\
Spontaneous breathing (high-frequency ventilation) & $1(3 \%)$ \\
Anesthesia conversion & $1(3 \%)$ \\
\hline
\end{tabular}



Figure $3 \mathrm{PaO}_{2}$ and $\mathrm{PaCO}_{2}$ during anesthesia $\left(\bar{x}_{ \pm}\right),{ }^{*} \mathrm{P}<0.05$ to after induction.

nerve palsy and recurrent laryngeal nerve paralysis (12). However, no clinical sign of phrenic nerve paralysis or recurrent laryngeal nerve paralysis were found in this study. The main reasons as follow: ultrasound technique applied to superficial CPB can improve the accuracy and effectiveness; there is a certain distance between the cervical plexus and the phrenic nerve/recurrent laryngeal nerve; the prevertebral fascia and carotid sheath have a substantial role in preventing the diffusion of local anesthetic; although the local anesthetic spreads to the deep cervical plexus, the concentration of the local anesthetic acting on the phrenic nerve and the recurrent laryngeal nerve is low; ropivacaine has a separation of motor sensation at low concentrations, unlikely to cause paralysis of the phrenic nerve or recurrent laryngeal nerve; partial or early symptoms of phrenic nerve paralysis and recurrent laryngeal nerve paralysis may difficult to distinguish due to overlap with respiratory depression while sedation. In this study, no clinical symptom of phrenic nerve paralysis or recurrent laryngeal nerve paralysis was found, and spontaneous breathing resumed during procedure. There were no symptoms of cough, hoarseness, and dyspnea postoperatively. In addition, in order to avoid cough reflex, tracheal local anesthesia was performed before incising the trachea and fiberoptic bronchoscopy in every case.

Another concern is hypoxemia. In our experience, during cervical TRR, the patients are in a state of double lung spontaneous breathing, rather than one lung spontaneous breathing in lung surgery. Therefore, after the trachea is incised, most patients can maintain $\mathrm{PaO}_{2}$ higher than $100 \mathrm{mmHg}$ when the patients resumed steady spontaneous breathing, and the $\mathrm{PaO}_{2}$ even better than in lung surgery (13). In our study, there was one patient with emphysema. This patient can resumed steady spontaneous breathing before the airway opened. After the trachea was incised, a sterile catheter was inserted in the distal portion of the trachea. The $\mathrm{SpO}_{2}$ maintained $96-100 \%$ when the airway was opened. However, because the trachea is opened, when the $\mathrm{SpO}_{2}$ decreased, it is unable to assisted ventilation temporarily like the lung surgery. Jet ventilation or even surgical cross-field intubation is needed when hypoxemia exist. In this study, after the trachea was incised, a sterile catheter was inserted in the distal trachea across the surgical field to increase fraction of inspired oxygen in all patients. $83 \%$ of patients maintained $\mathrm{SpO}_{2}$ higher than 90\%; 4 patients maintained $\mathrm{SpO}_{2}$ higher than $90 \%$ after removed the catheter and inhaled air only; in one case, although spontaneous breathing was observed after trachea 
was opened, $\mathrm{SpO}_{2}$ gradually decreased to $85 \%$, and jet ventilation was applied immediately. The $\mathrm{SpO}_{2}$ increased higher than $90 \%$ without anesthesia conversion. In one case, $\mathrm{SpO}_{2}$ suddenly decreased when dissecting trachea, diagnosed as right pleural rupture, immediate intubation across the surgical field. The patient resumed spontaneous breathing and the endotracheal tube was removed after treatment. It should be emphasized that the premise of maintaining stable oxygenation is to keep the airway clean and unobstructive. In addition, due to minute ventilation volume decreased when sedation we found out that there was mild hypercapnia in the cervical TRR. Hypercapnia has negative hemodynamic effects, for instance, arrhythmic and impaired cardiac contractility (14). None of the patients in our study showed any signs of hemodynamic instability. It was interesting that the $\mathrm{PaCO}_{2}$ slightly decreased after the airway is opened. We consider it's because the incision was first made distal of the lesion could relieve of obstruction.

LMA is a supraglottic airway devices has some advantages in tracheal surgery. It is a safe and wellestablished airway device without using muscle relaxant, obviates the complications of endotracheal intubation. LMA is beneficial to the patients with subglottic stenosis. LMA provides two conduits, one for ventilation as well as flexible bronchoscopy to assess the location and extent of the lesion during procedure, another for insertion of gastric tube to reduce the risk of reflux. During SBA for cervical tracheal surgery, without endotracheal tube obstructing recovery phase is a potentially risky period. After the surgery, a chin stitch would be used to keep the neck in flexion to decrease the tension on the anastomosis. Patients have better tolerance to the LMA, reducing the rates of coughing or head movement. In addition, the analgesia of the superficial $\mathrm{CPB}$ can last until the recovery phase, the patients were more comfortable, safer during recovery period.

In conclusion, according to our experience, SBA for cervical TRR is feasible. It can be an alternative anesthetic technique for cervical TRR in certain patients.

\section{Acknowledgments}

None.

\section{Footnote}

Conflicts of Interest: The authors have no conflicts of interest to declare.
Ethical Statement: The authors are accountable for all aspects of the work in ensuring that questions related to the accuracy or integrity of any part of the work are appropriately investigated and resolved. The study was approved by The First Affiliated Hospital of Guangzhou Medical University (No. 2015K12).

\section{References}

1. Liu J, Cui F, Li S, et al. Nonintubated Video-Assisted Thoracoscopic Surgery Under Epidural Anesthesia Compared With Conventional Anesthetic Option: A Randomized Control Study. Surg Innov 2015;22:123-30.

2. Vanni G, Tacconi F, Sellitri F, et al. Impact of Awake Videothoracoscopic Surgery on Postoperative Lymphocyte Responses. Ann Thorac Surg 2010;90:973-8.

3. Grillo HC, Zannini P, Michelassi F. Complication of tracheal reconstruction: Incidence, treatment, and prevention. J Thorac Cardiovasc Surg 1986;91:322-8.

4. Hobai IA, Chhangani SV, Alfille PH. Anesthesia for Tracheal Resection and Reconstruction. Anesthesiol Clin 2012;30:709-30.

5. Jiao D, Han X, Wu G, et al. Awake emergency endotracheal intubation using sheath-assisted technique for patients having malignant tracheal stenosis under fluoroscopy guidance: A retrospective study. Acta Radiol 2017;58:430-4.

6. Schieren M, Egyed E, Hartmann B, et al. Airway Management by Laryngeal Mask Airways for Cervical Tracheal Resection and Reconstruction: A Sin gle-Center Retrospective Analysis. Anesth Analg 2018;126:1257-61.

7. Stoelben E, Koryllos A, Beckers F, et al. Benign Stenosis of the Trachea. Thorac Surg Clin 2014;24:59-65.

8. Tsai TM, Lin MW, Hsu HH, et al. Nonintubated uniportal thoracoscopic wedge resection for early lung cancer. J Vis Surg 2017;3:155.

9. Pompeo E, Mineo D, Rogliani P, et al. Feasibility and Results of Awake Thora coscopic Resection of Solitary Pulmonary Nodules. Ann Thorac Surg 2004;78:1761-8.

10. Li S, Cui F, Liu J, et al. Nonintubated uniportal videoassisted thoracoscopic surgery for primary spontaneous pneumothorax. Chin J Cancer Res 2015;27:197-202.

11. Macchiarini P, Rovira I, Ferrarello S. Awake Upper Airway Surgery. Ann Thorac Surg 2010;89:387-90; discussion 390-1.

12. Sait Kavaklı A, Kavrut Öztürk N, Umut Ayoğlu R, et al. Comparison of Com bined (Deep and Superficial) and 
Intermediate Cervical Plexus Block by Use of Ultrasound Guidance for Carotid Endarterectomy. J Cardiothorac Vasc Anesth 2016;30:317-22.

13. Qinglong D, Lixia L, Yingfen L, et al. Anesthesia with nontracheal intubation in thoracic surgery. J Thorac Dis

Cite this article as: Zhou Y, Liu H, Wu X, Li S, Liang L, Dong Q. Spontaneous breathing anesthesia for cervical tracheal resection and reconstruction. J Thorac Dis 2019;11(12):5336-5342. doi: $10.21037 /$ jtd.2019.11.70
2012;4:126-30.

14. O'Croinin D, Ni Chonghaile M, Higgins B, et al. Benchto-bedside review: Per missive hypercapnia. Crit Care 2005;9:51-9. 